\title{
Étude Phytochimique et activité antifongique d'extraits de quelques Euphorbiaceae médicinales utilisées chez les Baoulé du District de Yamoussoukro (Côte d'Ivoire)
}

\author{
Saraka Allou Isidore \\ Laboratoire de Botanique, UFR Biosciences, \\ Université Félix Houphouët-Boigny, Abidjan, Côte d'Ivoire \\ Abo Kouabenan \\ Laboratoire de Phytopathologie et de Biologie Végétale, \\ Institut National Polytechnique Félix Houphouët-Boigny (INP-HB), \\ Yamoussoukro, Côte d'Ivoire \\ Coulibaly Kiyinlma \\ Faculté des Sciences Biologiques, \\ Université Péléforo Gon Coulibaly, Korhogo, Côte d'Ivoire \\ Zirihi Guédé Noël \\ Laboratoire de Botanique, UFR Biosciences, \\ Université Félix Houphouët Boigny, Abidjan, Côte d'Ivoire
}

Doi: 10.19044/esj.2018.v14n30p256 URL:http://dx.doi.org/10.19044/esj.2018.v14n30p256

\begin{abstract}
An ethnobotanical survey was carried out in the District of Yamoussoukro (Central Côte d'Ivoire) in order to evaluate the biocontrol potential against fungal plant pathogens, of Euphorbiaceous plants used as medicines by the native population. Twelve (12) species were collected. The antifungal activity was assessed using the two-fold dilution method on PDA medium with ethanolic and aqueous extract derived from leaves of six plants. Results pointed out that only $70 \%$ ethanolic extracts from Alchornea cordifolia $\left(\mathrm{EE}_{13}\right)$ possess fungicidal properties on both Fusarium sp. and Phytophthora sp. The antifungal activities are more effective with ethanolic extracts than aqueous extracts. With a minimal inhibitory concentration MIC $=25 \pm 0.0 \mathrm{mg} / \mathrm{ml}$, a minimal concentration for $50 \%$ of inhibition $\mathrm{IC}_{50}=$ $10 \pm 0.05 \mathrm{mg} / \mathrm{ml}$ and a minimal fungicide concentration MFC $=200 \pm 0.0$ $\mathrm{mg} / \mathrm{ml}$ Fusarium sp. appears to be the most sensitive. Phytophthora sp. is less sensitive with a MIC $=50 \pm 0.0 \mathrm{mg} / \mathrm{ml}$, an $\mathrm{IC}_{50}=12 \pm 0.05 \mathrm{mg} / \mathrm{ml}$ and a MFC $=200 \pm 0.0 \mathrm{mg} / \mathrm{ml}$. Phytochemical screening revealed the presence of seven major groups of compounds among which saponins and catechin are the most abundant in aqueous extracts. Polyphenols, flavonoids, polyterpenes and
\end{abstract}


sterols as well as tannins are present in both alcoholic and aqueous extracts of Alchornea cordifolia. These compounds could justify its antifungal activity and traditional use.

Keywords: Euphorbiaceae, Phytochemical Screening, Antifungal Activity, Alchornea cordifolia, Yamoussoukro District

\section{Résumé}

Une enquête ethnobotanique a été menée dans le district de Yamoussoukro (centre de la Côte d'Ivoire) afin d'évaluer le potentiel de lutte biologique contre les champignons pathogènes, de plantes de la famille des Euphorbiaceae utilisées à des fins médicinales par la population autochtone. Douze (12) espèces ont été collectées. L'activité antifongique a été évaluée par la méthode de double dilution sur milieu PDA avec des extraits éthanoliques et aqueux dérivés d'organe de six plantes. Les résultats ont montré que seuls des extraits éthanoliques $70 \%$ d'Alchornea cordifolia $\left(\mathrm{EE}_{13}\right)$ possèdent des propriétés fongicides à la fois sur Fusarium sp. et Phytophthora sp. Les activités antifongiques sont plus importantes avec les extraits éthanoliques que les extraits aqueux. Avec une concentration minimal inhibitrice CMI $=25 \pm 0,0$ $\mathrm{mg} / \mathrm{ml}$, une $\mathrm{CI}_{50}=10 \pm 0,05 \mathrm{mg} / \mathrm{ml}$ et une concentration minimal fongicide CMF $=200 \pm 0,0 \mathrm{mg} / \mathrm{ml}$ Fusarium sp. semble être le plus sensible. Phytophthora sp. est moins sensible avec une CMI $=50 \pm 0,0 \mathrm{mg} / \mathrm{ml}$, une $\mathrm{CI}_{50}$ $=12 \pm 0,05 \mathrm{mg} / \mathrm{ml}$ et une $\mathrm{CMF}=200 \pm 0,0 \mathrm{mg} / \mathrm{ml}$. Le screening phytochimique a mis en évidence la présence de sept grands groupes de composés parmi lesquels les saponines et les catéchines sont les plus abondants dans les extraits aqueux. Les Polyphénols, les Flavonoïdes, les Polyterpènes et Stérols ainsi que les Tanins sont aussi bien présents dans les extraits alcooliques que les extraits aqueux d'Alchornea cordifolia. Ces composés pourraient justifier son activité et son utilisation traditionnelle.

Mots clés: Euphorbiaceae, Screening phytochimique, Activité antifongique, Alchornea cordifolia, District de Yamoussoukro

\section{Introduction}

L'agriculture est la base de l'économie de nombreux pays de l'Afrique subsaharienne (Soro et al., 2012). Cependant celle-ci est menacée de nombreuses espèces parasites fongiques dont Phytophthora sp. et Fusarium $s p$. Ces phytopathogènes causent des dégâts aussi bien au niveau des cultures de rente, des cultures vivrières que des fruits tropicaux comme l'avocat, la mangue, la banane, la papaye, la tomate, et le cotonnier (Hibar et al., 2007; Soro et al., 2012). Fusarium sp. sont aussi à l'origine de nécroses importantes observées sur les fruits entreposés après les récoltes (Kouadia, 2010). Par 
ailleurs Phytophthora sp. agent causatif des pourritures noire et brune crée de nombreux dégâts chaque année allant jusqu'à la perte de la totalité de la production de fèves de cacao dans certaines pays (Flood, 2006). Face à cette situation les agriculteurs ont recours aux fongicides pour la protection de leur culture ou leur récolte. Malheureusement l'utilisation des pesticides de synthèse n'est pas sans danger sur la santé des agricultures et des consommateurs (de Jaeger, 2012). En outre, les pays importateurs des fruits tropicaux sont de plus en plus exigeants quant aux résidus de pesticides et autres contaminants (Pinon, 2000). Il est donc important d'explorer d'autres voies pour la protection des cultures, de l'environnement et la préservation de la santé des acteurs de la filière.

L'utilisation des substances naturelles extraites de plantes pourrait constituer une alternative sérieuse aux fongicides chimiques. En effet, au Maroc, l'activité antifongique de Thymelaea lythroides contre Curvularia lunata, Helminthosporium oryzae et Pyricularia oryzae, trois champignons pathogènes du Riz a été démontré (Dohou et al., 2004). Au Cameroun, l'activité antifongique de Citrus latifolia contre Phaeoramularia angolensis, un champignon pathogène infectant les agrumes a été révélé par Jazet et al. (2008). En Côte d'Ivoire, Kra et al., (2009) et Soro et al., (2010) ont respectivement établi l'efficience anti-fusariose de Chromolaena odorata et de Xylopia aethiopica (Dunal) A. Rich. Une étude a été menée à la fois in vitro et in vivo afin d'évaluer l'activité antifongique de l'huile essentielle d'Ocimum gratissimum L. sur Fusarium oxysporum f. sp. radicis-lycopersici (Soro et al., 2011).

C'est dans ce cadre que notre étude ethnobotanique conduite dans le District de Yamoussoukro a permis d'identifier 12 Euphorbiaceae utilisées à des fins médicinales par le peuple autochtone Baoulé de cette circonscription (Saraka et al., 2018). La présente étude a pour objectif d'évaluer le potentiel antifongique des extraits aqueux et éthanoliques de quelques-unes de ces plantes.

\section{Matériel Et Méthodes D'étude}

Materiel

\section{Matériel végétal}

Le matériel végétal est constitué de douze espèces de la famille des Euphorbiaceae récoltés dans le District de Yamoussoukro (Tableau I). 
Tableau I : Nature des organes prélevés pour la réalisation des poudres de plantes

\begin{tabular}{|c|c|c|c|}
\hline $\mathrm{N}^{\circ}$ & Noms scientifiques & Codes & Organes prélevés \\
\hline & & $\mathrm{P}_{1}$ & Feuilles fraîches \\
\cline { 3 - 4 } 1 & Alchornea cordifolia & $\mathrm{P}_{13}$ & Feuilles mortes \\
\hline 2 & Croton hirtus & $\mathrm{P}_{2}$ & Rameaux feuillés \\
\hline 3 & Croton lobatus & $\mathrm{P}_{3}$ & Rameaux feuillés \\
\hline 4 & Elaeophorbia grandifolia & $\mathrm{P}_{4}$ & Feuilles fraîches \\
\hline 5 & Euphorbia heterophylla & $\mathrm{P}_{5}$ & Rameaux feuillés \\
\hline 6 & Euphorbia hirta & $\mathrm{P}_{6}$ & Rameaux feuillés \\
\hline 7 & Euphorbia hypericifolia & $\mathrm{P}_{7}$ & Rameaux feuillés \\
\hline 8 & Jatropha curcas & $\mathrm{P}_{8}$ & Feuilles fraîches \\
\hline 9 & Jatropha gossypiifolia & $\mathrm{P}_{9}$ & Feuilles frâ̂ches \\
\hline 10 & Manihot esculenta & $\mathrm{P}_{10}$ & Feuilles frâ̂ches \\
\hline 11 & Ricinodendron heudelotii & $\mathrm{P}_{11}$ & Ecorce de tige \\
\hline 12 & Ricinus communis & $\mathrm{P}_{12}$ & Feuilles frâ̂ches \\
\hline
\end{tabular}

\section{Matériel fongique}

Le matériel fongique est constitué de Phytophthora sp. et Fusarium sp. Ces souches ont été fournies par le Laboratoire de Phytopathologie et de Biologie Végétale de l'Institut National Polytechnique Félix HouphouëtBoigny de Yamoussoukro.

\section{Methodes}

\section{Récolte et séchage des organes végétaux}

Les organes de plantes de la famille des Euphorbiaceae ont été récoltés entre Mai et Août 2017 à la suite d'une enquête ethnobotanique dans cinq villages du District de Yamoussoukro (Saraka et al., 2018). Ils ont été lavés à l'eau de robinet, découpés et séchés à l'ombre dans une salle à la température ambiante $\left(26^{\circ} \mathrm{C}\right)$ et à $1^{\prime}$ 'abri du soleil pendant 21 jours.

\section{Préparation des extraits aqueux et éthanolique}

Les organes de plante initialement séchés ont été pulvérisés grâce à un broyeuse électrique de type Grinder GLG-450. La fine poudre obtenue a subi une extraction selon la méthode de Zirihi et al., (2003), ainsi décrite :

Cent grammes $(100 \mathrm{~g})$ de poudre d'organe végétal ont été mélangés, dans un litre (1L) d'eau distillée grâce à un mixeur de type Blender, trois fois pendant trois minutes. L'homogénat obtenu est d'abord essoré dans un carré de tissu pour éliminer les débris végétaux, puis filtré successivement deux fois sur du coton hydrophile pour éliminer les débris qui ont pu traverser les mailles du tissu. Enfin, une dernière filtration a été faite une fois sur du papier filtre Whatman. Le filtrat obtenu est séché à l'étuve à $50^{\circ} \mathrm{C}$ pendant cinq jours. La poudre ainsi obtenue est l'extrait total aqueux noté ETA. Ce même procédé a 
été utilisé pour chacune des douze espèces végétales recensées. Ces extraits sont nommés ETA1 à ETA12 correspondant respectivement aux codes P1 à P12. Un extrait nommé ETA13 a été produit à partir des feuilles mortes d 'A. cordifolia qui sont généralement utilisés comme telles pour les soins de santé. Tous les extraits ont été ensuite mis dans des récipients stériles puis fermés hermétiquement et conservés au réfrigérateur à $6{ }^{\circ} \mathrm{C}$ jusqu'à utilisation.

L'extrait éthanolique a été obtenu de trois plantes selon la deuxième variante de la méthode de Zirihi et al. (2003). L'extraction s'est faite de manière similaire que l'extrait aqueux mais avec comme solvant l'éthanol $70 \%$. Les extraits éthanoliques ont été codés $\mathrm{EE}_{13}, \mathrm{EE}_{10}, \mathrm{EE}_{12}$ et conservés dans les mêmes conditions que les extraits aqueux. Tous les extraits obtenus ont été pesés pour l'évaluation du rendement de l'extraction.

\section{Calcul du rendement}

Le rendement est déterminé par le rapport de la masse de l'extrait sec après évaporation sur la masse de la poudre de matière végétale sèche utilisée pour l'extraction. Il est exprimé en pourcentage et est calculé par la formule suivante :

$$
\mathbf{R d}=(\mathbf{m} \times \mathbf{1 0 0}) / \mathrm{M} \text {; }
$$

Où Rd désigne le rendement d'extraction en pourcentage, $\mathbf{m}$, la masse en gramme de l'extrait sec et, $\mathbf{M}$ la masse en gramme de la poudre d'organe végétal.

\section{Screening phytochimique}

Les grandes familles de métabolites secondaires ont été recherchées dans les extraits (aqueux et hydro alcooliques) de plantes suivant les méthodes classiques de caractérisation par réactions colorées (Mangambu et al., 2014). Les solutions présentant des réactions positives indiquent la présence des groupes chimiques mis en évidence dans l'extrait. Les résumés des réactions sont contenus dans le Tableau II.

Tableau II : Réactions d'identification des groupes chimiques et indicateurs utilisés

\begin{tabular}{|c|c|c|c|}
\hline \multicolumn{2}{|c|}{ Groupes chimiques } & Réactifs d'identification & Indicateur (Réaction positive) \\
\hline Stérols et polyterpènes & $\begin{array}{c}\text { Anhydride acétique, Acide } \\
\text { sulfurique concentré }\end{array}$ & $\begin{array}{c}\text { Apparition à l'interphase d'un anneau pourpre ou } \\
\text { violet, virant au bleu puis au vert. }\end{array}$ \\
\hline \multirow{2}{*}{ Polyphénols } & Chlorure ferrique FeCl3 (2\%) & $\begin{array}{c}\text { Apparition d'une coloration bleue noirâtre ou } \\
\text { verte plus ou moins foncée }\end{array}$ \\
\hline \multicolumn{2}{|c|}{ Flavonoïdes } & $\begin{array}{c}\text { Alcool chlorhydrique, Copaux de } \\
\text { Magnésium, Alcool iso-amylique }\end{array}$ & $\begin{array}{c}\text { Dégagement de chaleur puis coloration rose- } \\
\text { orange ou violacée }\end{array}$ \\
\hline \multirow{2}{*}{ Tanins } & Catéchiques & $\begin{array}{c}\text { Formaldéhyde, Acide } \\
\text { chlorhydrique concentré }\end{array}$ & Précipité gélatineux (en gros flocons) \\
\cline { 2 - 5 } & Galliques & $\begin{array}{c}\text { Acétate de sodium, Chlorure } \\
\text { ferrique }\end{array}$ & Coloration bleue-noire intense \\
\hline \multicolumn{2}{|c|}{ Quinones } & Ammoniaque & Apparition d'une coloration allant du rouge au \\
violet.
\end{tabular}




\begin{tabular}{|c|c|c|}
\hline Saponosines & Indice de mousse & Apparition d'une mousse persistante \\
\hline \multirow{2}{*}{ Alcaloïdes } & $\begin{array}{c}\text { Dragendorff (Solution iodo- } \\
\text { bismuthate de potassium) }\end{array}$ & \multirow{2}{*}{ Précipité de coloration brun rougeâtre } \\
\cline { 2 - 2 } & Burchard (Reaction iodo-iodurée) & \\
\hline
\end{tabular}

\section{Test d'activité antifongique}

Les tests d'activité antifongique ont été réalisés sur des souches de Phytophthora sp. et Fusarium sp.

Un test antifongique préliminaire de tri a été effectué avec les extraits aqueux des 12 plantes sur Phytophthora sp. et Fusarium sp. selon le seuil d'efficacité $(25 \mathrm{mg} / \mathrm{ml})$ de Kra (2016). Ce test avait pour objectif de sélectionner les trois premières plantes dont les extraits aqueux inhiberaient à la fois Phytophthora sp. et Fusarium sp. Le milieu PDA (Potato Dextrose Agar) a été utilisé pour la culture des champignons. Une gamme de cinq concentrations a été retenue pour l'extrait aqueux (ETA) et pour l'extrait éthanolique $70 \%$ (EE). Ces concentrations sont : $100 \mathrm{mg} / \mathrm{ml}, 50 \mathrm{mg} / \mathrm{ml}, 25$ $\mathrm{mg} / \mathrm{ml}, 12,5 \mathrm{mg} / \mathrm{ml}$ et $6,25 \mathrm{mg} / \mathrm{ml}$. Elles ont été définies selon la méthode de la double dilution de liaison géométrique de raison $1 / 2$ (Zirihi et al., 2003). Les différentes quantités des extraits végétaux ont été incorporées aux milieux de culture dans des bouteilles de $500 \mathrm{ml}$ SCHOTT DURAN. Ces milieux ont été stérilisés à l'autoclave à $120^{\circ} \mathrm{C}$ pendant 20 minutes. Les témoins n'ont reçu aucun apport d'extraits. Après homogénéisation, les différents mélanges ont été coulés à $40{ }^{\circ} \mathrm{C}$ dans des boîtes de Pétri de $90 \mathrm{~mm}$ de diamètre, sous une hotte dans des conditions aseptiques autour d'une flamme. Un essai à trois répétitions a été effectué pour chaque concentration et chaque souche fongique. Les boîtes de Pétri ont été maintenues sous la hotte jusqu'à la solidification du milieu et à l'ensemencement du fragment mycélien. Un explant de $6 \mathrm{~mm}$ de diamètre de chaque souche fongique a été prélevé au niveau du front de croissance du champignon dans la boîte de culture. L'explant a été placé au centre de la boîte de Pétri sur le milieu solidifié. Les boîtes de Pétri ensemencées ont ensuite été scellées avec du film adhésif et mises en incubation à l'étuve pendant 24 heures à $25 \pm 2^{\circ} \mathrm{C}$. La mesure de la croissance radiale moyenne du mycélium des différentes souches fongiques a été effectuée quotidiennement parallèlement au témoin. Cette mesure s'est réalisée en millimètre à partir de deux axes perpendiculaires tracées au revers de la boîte de Pétri. Les diamètres du mycélium ont été mesurés jusqu'à ce que dans les lots témoins, les filaments mycéliens atteignent la périphérie de la boîte de Pétri. C'est au septième jour post ensemencement que la prise de mesure a été arrêtée. Le taux d'inhibition de la croissance radiale mycélienne a été calculé selon la formule de Leroux et Credet (1978).

$$
\mathbf{T}(\%)=(\mathbf{D}-\mathbf{d}) / \mathrm{D}) \times \mathbf{x} 100
$$

(T : taux d'inhibition, $\mathbf{D}$ : croissance mycélienne dans les boîtes de Pétri témoins, $\mathbf{d}$ : croissance mycélienne dans les boîtes essais). 
La détermination du taux d'inhibition de la croissance mycélienne de chaque souche fongique a permis de définir, pour chaque extrait, la concentration minimale inhibitrice (CMI) et la concentration minimale fongicide (CMF). La CMI correspond à la concentration qui donne $100 \%$ d'inhibition et la CMF correspond à la plus petite concentration à partir de laquelle aucune croissance mycélienne n'est observée dans les nouvelles boîtes de Pétri où les explants ont été réensemencées après avoir déterminé la CMI. La concentration qui donne 50\% du taux d'inhibition de la croissance des germes fongiques $\left(\mathrm{CI}_{50}\right)$ et la concentration qui donne $90 \%$ du taux d'inhibition de la croissance des germes fongiques ( $\left.\mathrm{CI}_{90}\right)$ ont été déterminées graphiquement (Ackah, 2004).

\section{Traitement et analyse statistique des données}

Le logiciel STATISTICA Version 7.1 a servi à l'ANOVA un facteur au seuil de 5\%. Les moyennes ont été classées selon le test LSD de Fisher. Les calculs numériques et la construction des graphiques ont été effectués avec le logiciel Excel.

\section{Résultats}

\section{Sélection des organes de plantes}

Les taux d'inhibition des treize extraits végétaux obtenus sur la croissance mycélienne de Phytophthora sp. ont varié de 57,13 $\pm 18,95 \%$ à 0,29 $\pm 3,68 \%$. La plus faible inhibition a été obtenue avec l'extrait de $\mathrm{P} 3(0,29 \pm$ $3,68 \%)$ et la plus forte avec l'extrait de P13 (57,13 $\pm 18,95 \%)$ voir Figures 1 et 2 . D'après l'analyse de la variance, cette différence est significative $(\mathrm{P}=0$ $<0,05)$.

Les taux d'inhibition des treize extraits végétaux obtenus sur la croissance mycélienne de Fusarium sp. ont varié de 56,86 $\pm 5,22 \%$ à $8,10 \pm$ $11,42 \%$. La plus faible inhibition a été obtenue avec l'extrait de P5 $(8,10 \pm$ $11,42 \%)$ et la plus forte avec l'extrait de P13 (56,86 $\pm 5,22 \%)$ voir Figures 1 et 2 . D'après l'analyse de la variance, cette différence est significative $(\mathrm{P}=0$ $<0,05)$.

Il ressort de cette analyse que les trois premiers extraits ayant des effets inhibiteurs à la fois sur Phytophthora sp. et Fusarium sp. sont P10, P12 et P13.

P10 a eu des taux d'inhibition de $33,25 \pm 9,45 \%$ et $38,64 \pm 10,09 \%$ respectivement sur Phytophthora sp. et Fusarium sp. P12 a eu des taux d'inhibition de $38,19 \pm 12,79 \%$ et $49,80 \pm 5,16 \%$ respectivement sur Phytophthora sp. et Fusarium sp. P13 a eu des taux d'inhibition de 57,13 \pm $18,95 \%$ et 56,86 $\pm 5,22 \%$ respectivement sur Phytophthora sp. et Fusarium $s p$. Les feuilles des plantes P10, P12 et P13 ont donc été sélectionnées pour la suite du travail. Les extraits ETA et EE de chaque plante ont été testés de manière dose dépendante sur la croissance mycélienne de Phytophthora sp. et 
Fusarium sp. Les feuilles mortes d'Alchornea cordifolia (P13) ont présenté les meilleurs paramètres antifongiques (Tableau III).
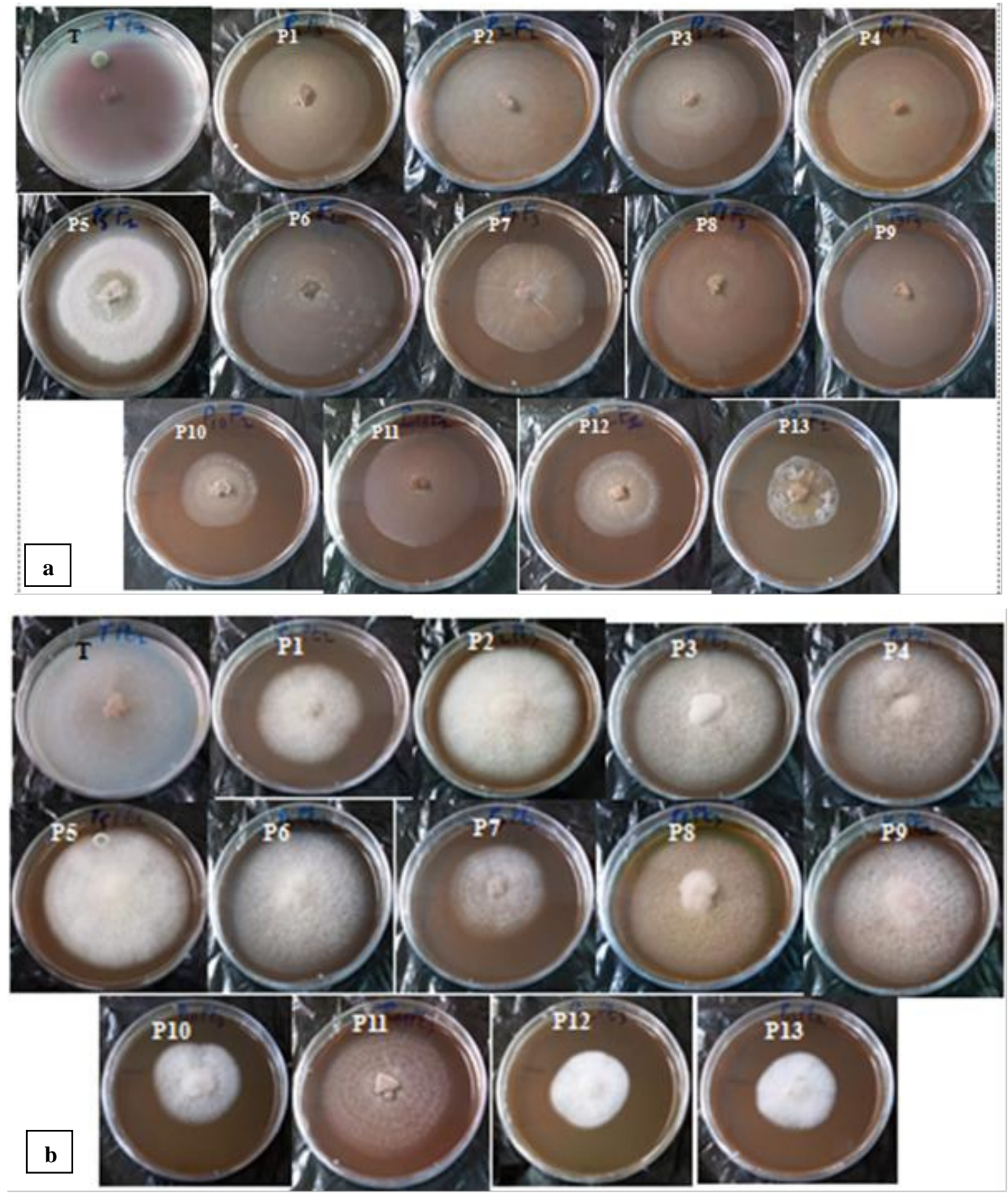

Figure 1 : Croissance in vitro du mycélium de Fusarium sp (a) et Phytophthora sp. (b) en présence de treize extraits aqueux d'organes de douze plantes à $\mathrm{C}=25 \mathrm{mg} / \mathrm{ml}$ et Témoin $(\mathrm{T})=0 \mathrm{mg} / \mathrm{ml}$ après 7 jours d'incubation 


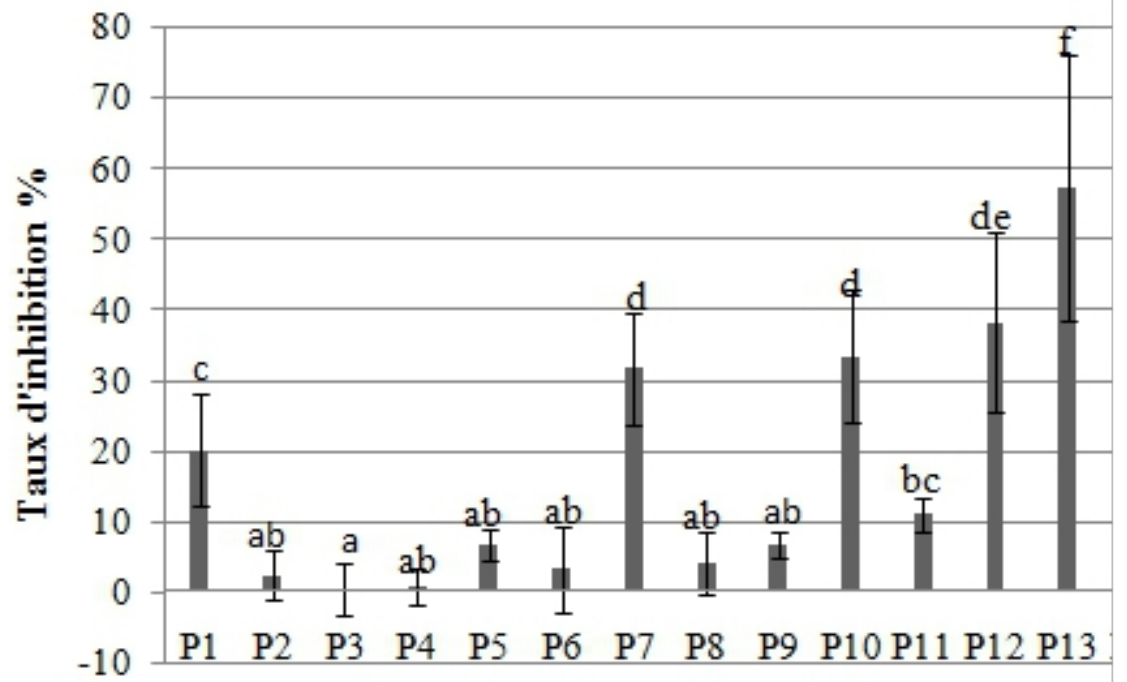

Plantes testées sur Phytophthora sp.

Figure 2a : Taux d'inhibition de la croissance mycélienne de Phytophthora sp. à la concentration de $25 \mathrm{mg} / \mathrm{ml}$ d'ETA de chaque plante

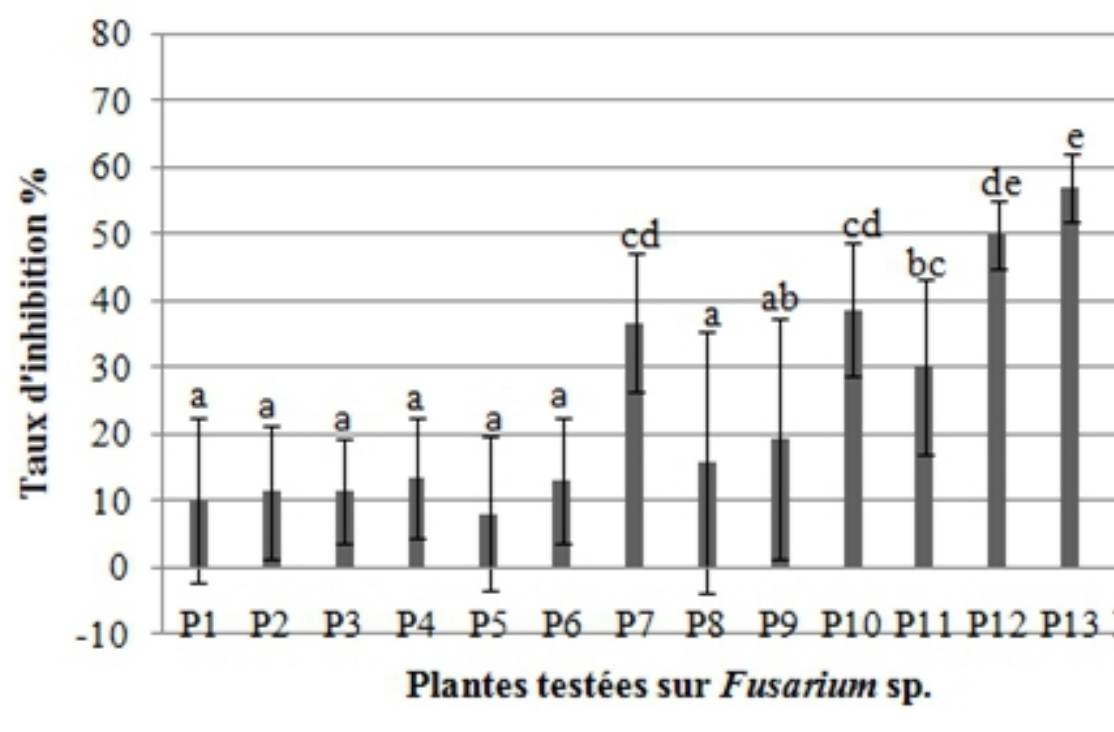

Figure 2b : Taux d'inhibition de la croissance mycélienne de Fusarium $s p$. à la concentration de $25 \mathrm{mg} / \mathrm{ml}$ d'ETA de chaque plante 
Tableau III : Paramètres antifongiques des extraits ETA et EE des trois plantes

\begin{tabular}{|c|c|c|c|c|c|c|c|c|c|}
\hline \multirow[t]{2}{*}{ Phytopathogènes } & \multirow{2}{*}{$\begin{array}{l}\text { Extrait } \\
\text { végétal }\end{array}$} & \multicolumn{2}{|c|}{ CI50 (mg/ml) } & \multicolumn{2}{|c|}{ CI90 (mg/ml) } & \multicolumn{2}{|c|}{$\begin{array}{c}\mathrm{CMI} \\
(\mathrm{mg} / \mathrm{ml})\end{array}$} & \multicolumn{2}{|c|}{$\begin{array}{c}\text { CMF } \\
(\mathrm{mg} / \mathrm{ml})\end{array}$} \\
\hline & & ETA & $\mathrm{EE}(70 \%)$ & ETA & $\mathrm{EE}(70 \%)$ & ETA & $\mathrm{EE}(70 \%)$ & ETA & $\mathrm{EE}(70 \%)$ \\
\hline \multirow{3}{*}{ Fusarium sp. } & $\mathbf{P 1 3}$ & 26 & 10 & 86 & 22 & 100 & 25 & 200 & 200 \\
\hline & P10 & 5 & $>50$ & $>50$ & $>100$ & $>100$ & $>100$ & ND & ND \\
\hline & $\mathrm{P} 12$ & $<6$ & 44 & NA & ND & NA & $>100$ & NA & ND \\
\hline \multirow{3}{*}{ Phytophthora sp. } & $\mathbf{P 1 3}$ & 19 & 12 & 91 & 41 & $>100$ & 50 & 200 & 200 \\
\hline & $\mathrm{P} 10$ & 19 & $>50$ & $>50$ & $>50$ & $>100$ & $>100$ & $\mathrm{ND}$ & ND \\
\hline & $\mathrm{P} 12$ & 21 & 36 & NA & ND & NA & $>100$ & NA & ND \\
\hline
\end{tabular}

\section{Screening phytochimique}

Le screening phytochimique a été effectué aussi bien pour les extraits aqueux que les extraits éthanoliques des trois plantes retenues.

Extrait aqueux: Le screening phytochimique des espèces collectées (Tableau IVa) a révélé une forte présence de saponines, de tanins et de polyphénols dans tous les extraits, excepté l'extrait de Ricinus communis (ETA12) qui ne contient pas de tanins. Le screening a aussi révélé la présence de polyterpènes dans les différents extraits (ETA13 et ETA10) sauf dans les extraits de Ricinus communis (ETA12). Il a mis en évidence également la présence d'alcaloïdes uniquement dans les extraits de feuilles d'Alchornea cordifolia (ETA13). Les flavonoïdes sont présents uniquement dans les extraits de feuilles d'Alchornea cordifolia. Cependant aucun extrait ne contenait des quinones.

Tableau IVa: Métabolites secondaires identifiés dans les extraits aqueux

\begin{tabular}{|c|c|c|c|c|c|c|c|c|}
\hline $\begin{array}{c}\text { Métabolite } \\
\text { secondaire }\end{array}$ & Saponine & Polyphénol & Flavonoïde & $\begin{array}{c}\text { Polyterpène } \\
\text { et stérol }\end{array}$ & Alcalö̈d & $\begin{array}{c}\text { Tanin } \\
\text { gallique }\end{array}$ & $\begin{array}{c}\text { Tanin } \\
\text { catéchique }\end{array}$ & Quinone \\
\hline $\begin{array}{c}\text { Alchornea } \\
\text { Cordifolia }\end{array}$ & +++ & + & + & + & + & ++ & ++ & - \\
\hline $\begin{array}{c}\text { Manihot } \\
\text { esculenta }\end{array}$ & + & + & - & + & - & - & ++ & - \\
\hline $\begin{array}{c}\text { Ricinus } \\
\text { communis. }\end{array}$ & ++ & + & - & - & - & - & - & - \\
\hline
\end{tabular}

-: Absent, +: Présent; ++: Abondant; +++: Très Abondant

Extrait éthanolique: Le criblage phytochimique des extraits éthanoliques des organes de plantes collectées présente des résultats presque similaires à ceux des extraits aqueux à la grande différence d'une réaction négative au test de saponine avec tous les extraits. De plus tous les extraits hydro-éthanoliques contiennent des stérols et polyterpènes. En outre l'extrait de Ricinus communis (EE12) contient des tannins galliques (Tableau IVb). 
Tableau IVb: Métabolites secondaires identifiés dans les extraits éthanoliques

\begin{tabular}{|c|c|c|c|c|c|c|c|c|}
\hline $\begin{array}{c}\text { Métabolite } \\
\text { secondaire }\end{array}$ & Saponine & Polyphénol & Flavonö̈de & $\begin{array}{c}\text { Polyterpène } \\
\text { et stérol }\end{array}$ & Alcaloïde & $\begin{array}{c}\text { Tanin } \\
\text { gallique }\end{array}$ & $\begin{array}{c}\text { Tanin } \\
\text { catéchique }\end{array}$ & Quinone \\
\hline $\begin{array}{c}\text { Alchornea } \\
\text { Cordifolia }\end{array}$ & - & + & + & + & + & + & + & - \\
\hline $\begin{array}{c}\text { Manihot } \\
\text { esculenta }\end{array}$ & - & + & - & + & - & - & ++ & - \\
\hline $\begin{array}{c}\text { Ricinus } \\
\text { communis. }\end{array}$ & - & + & - & + & - & ++ & - & - \\
\hline
\end{tabular}

-: Absent, +: Présent; ++: Abondant; +++: Très Abondant

\section{Rendement des extraits}

Les extractions aqueuse et hydro éthanolique $70 \%$ directement sur la drogue ont permis d'obtenir les différents rendements consignés dans le Tableaux V. Ils varient de $14,075 \pm 0,025$ à $27,16 \pm 0,16 \%$ pour les extraits aqueux et de 19,28 $\pm 0,29$ à $32,36 \pm 0,06 \%$ pour les extraits éthanoliques. Les feuilles de Manihot esculenta ont donné le plus faible rendement à la fois par leur extrait aqueux ETA10 $(14,075 \pm 0,025)$ que par leur extrait hydroéthanolique $70 \% \mathrm{EE}_{10}(19,28 \pm 0,29)$. Les feuilles de Ricinus communis ont donné le meilleur rendement aussi bien par leur extrait aqueux ETA12 (27,16 $\pm 0,16 \%)$ que par leur extrait hydro-éthanolique $70 \% \mathrm{EE}_{12}(32,36 \pm 0,06 \%)$.

Tableau V : Rendement des extraits ETA et EE des trois plantes testées

\begin{tabular}{|c|c|c|c|c|}
\hline \multicolumn{2}{|c|}{ Espèces végétales (drogues utilisées) } & $\begin{array}{c}\text { Masse } \\
\text { poudre }(\mathbf{g})\end{array}$ & $\begin{array}{c}\text { Rendement } \\
\text { ETA }(\%)\end{array}$ & $\begin{array}{c}\text { Rendement EE70\% } \\
(\%)\end{array}$ \\
\hline $\mathrm{P}_{13}$ & $\begin{array}{c}\text { Alchornea cordifolia } \text { (Schum. } \\
\text { \& Thonn.) Müll.Arg. }\end{array}$ & 100 & $27,06 \pm 1,9$ & $23,2 \pm 0,48$ \\
\hline $\mathrm{P}_{10}$ & Manihot esculenta Crantz & 100 & $14,075 \pm 0,025$ & $19,28 \pm 0,29$ \\
\hline $\mathrm{P}_{12}$ & Ricinus communis Linn. & 100 & $27,16 \pm 0,16$ & $32,36 \pm 0,06$ \\
\hline
\end{tabular}

\section{Taux d'inhibition de la croissance mycélienne}

Les observations de l'effet des extraits aqueux et éthanoliques sur la croissance mycélienne des souches Phytophthora sp. et Fusarium sp. ont révélé comparativement au témoin, une diminution de la croissance mycélienne au fur et à mesure que les concentrations des extraits ETA et EE augmentent dans les boîtes de pétri (Figure 3 et 6). 


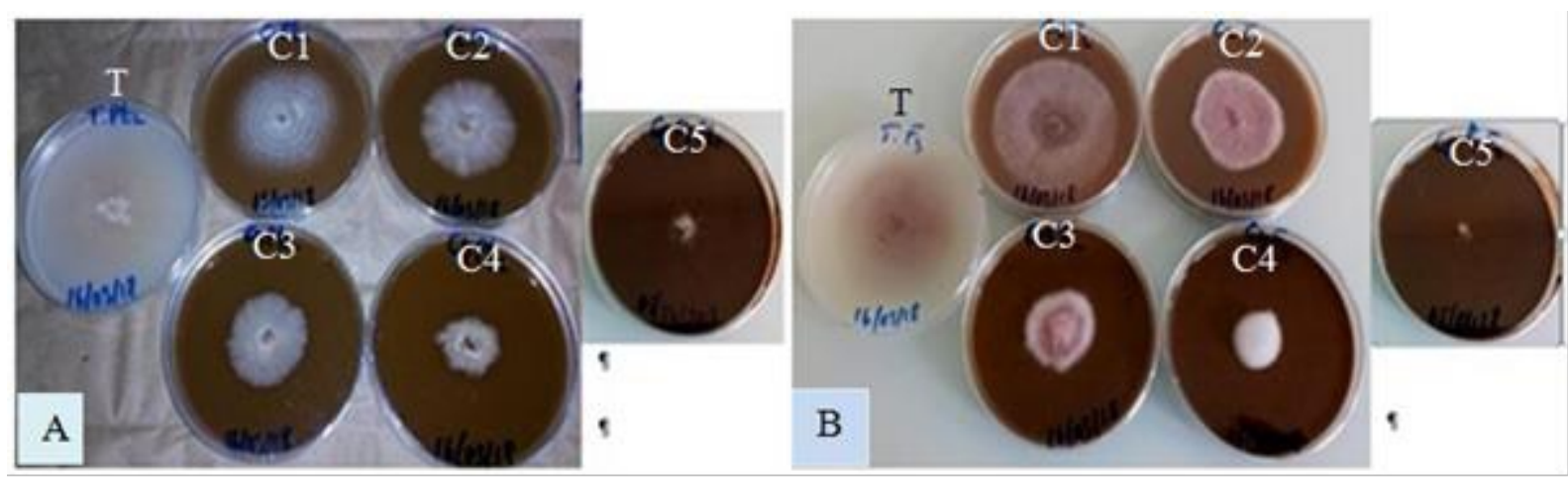

Figure 3 : Croissance in vitro du mycélium de Phytophthora sp. (A) et Fusarium sp (B) en présence d'extrait aqueux de feuilles d'Alchornea cordifolia (ETA13) après 7 jours d'incubation

T : Témoin $0 \mathrm{mg} / \mathrm{ml} ; \mathrm{C} 1: 6,25 \mathrm{mg} / \mathrm{ml} ; \mathrm{C} 2: 12,5 \mathrm{mg} / \mathrm{ml} ; \mathrm{C} 3: 25 \mathrm{mg} / \mathrm{ml} ; \mathrm{C} 4: 50 \mathrm{mg} / \mathrm{ml}$; C5 : $100 \mathrm{mg} / \mathrm{ml}$

Le mycélium atteint la périphérie de la boîte dans les témoins le 7ème jour d'incubation pour les deux types d'extraits. Les deux phytopathogènes étudiés n'ont pas la même sensibilité vis à vis des extraits d'Alchornea cordifolia testés. Avec l'extrait aqueux, les plus forts taux d'inhibition de 94.98 $\%$ et $100 \%$, ont respectivement été obtenus avec la concentration de 100 $\mathrm{mg} / \mathrm{ml}$ pour Phytophthora sp. et $100 \mathrm{mg} / \mathrm{ml}$ pour Fusarium sp. (Figures 4 et $5)$.

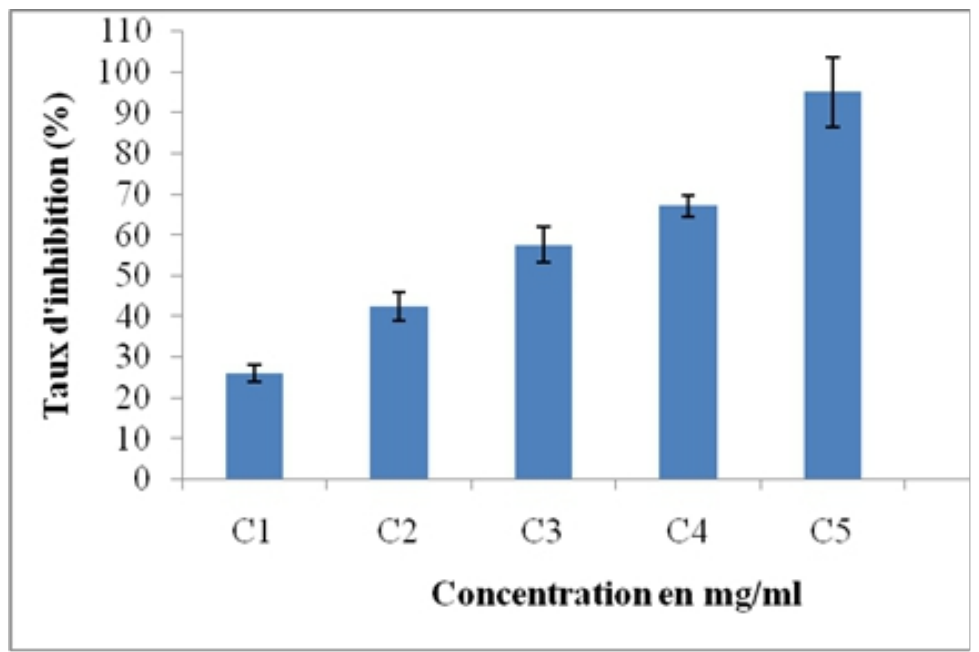

Figure 4: Taux d'inhibition de la croissance mycélienne de Phytophthora sp. en fonction de la concentration d'ETA13 


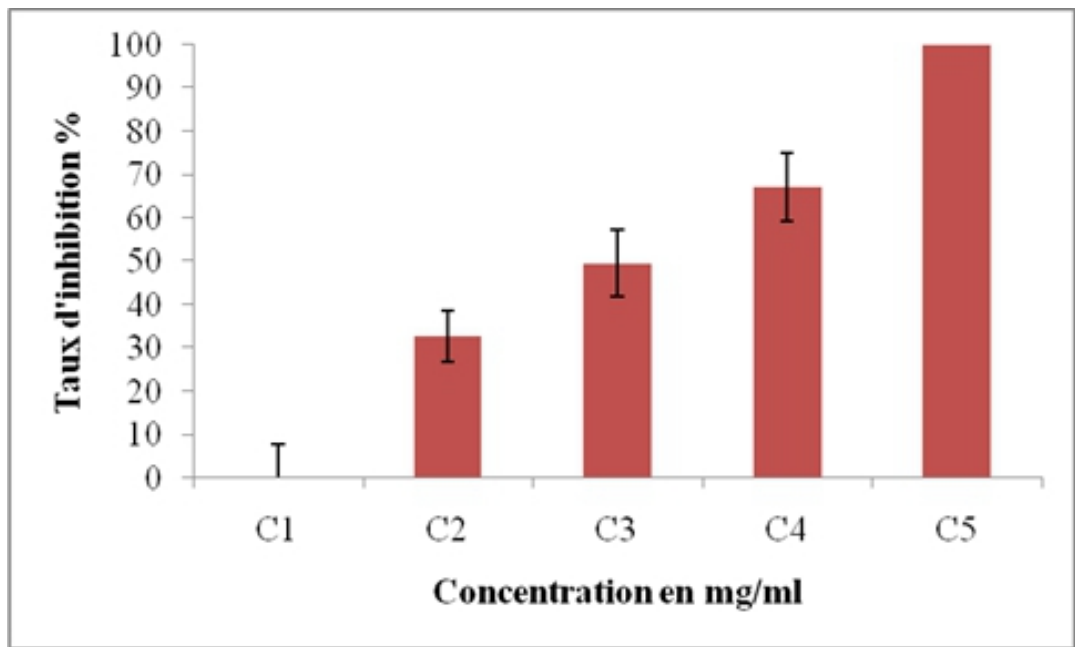

Figure 5: Taux d'inhibition de la croissance mycélienne de Fusarium sp. en fonction de la concentration d'ETA13

Par ailleurs, avec l'extrait éthanolique, aucune croissance mycélienne n'a été observée chez Fusarium sp. à la concentration de $25 \mathrm{mg} / \mathrm{ml}$, de même aucune croissance n'a été observée aussi bien chez Fusarium $s p$. que chez Phytophthora sp. à la concentration de $50 \mathrm{mg} / \mathrm{ml}$ (Figures 6, 7 et 8).
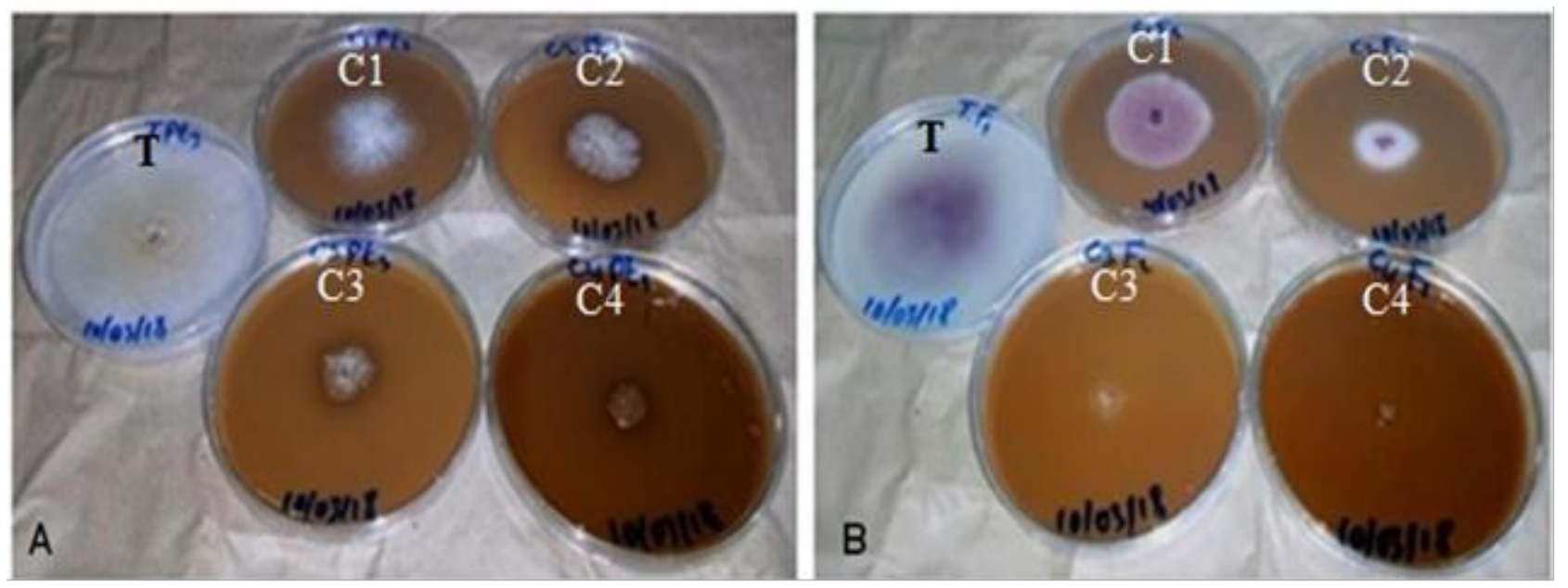

Figure 6 : Croissance in vitro du mycélium de Phytophthora sp. (A) et Fusarium sp (B) en présence d'extrait éthanolique de feuilles d'Alchornea cordifolia (EE13) après 7 jours d'incubation

T : Témoin 0 mg/ml ; C1 : 6,25 mg/ml ; C2 : 12,5 mg/ml; C3 : $25 \mathrm{mg} / \mathrm{ml}$; C4 : $50 \mathrm{mg} / \mathrm{ml}$. 


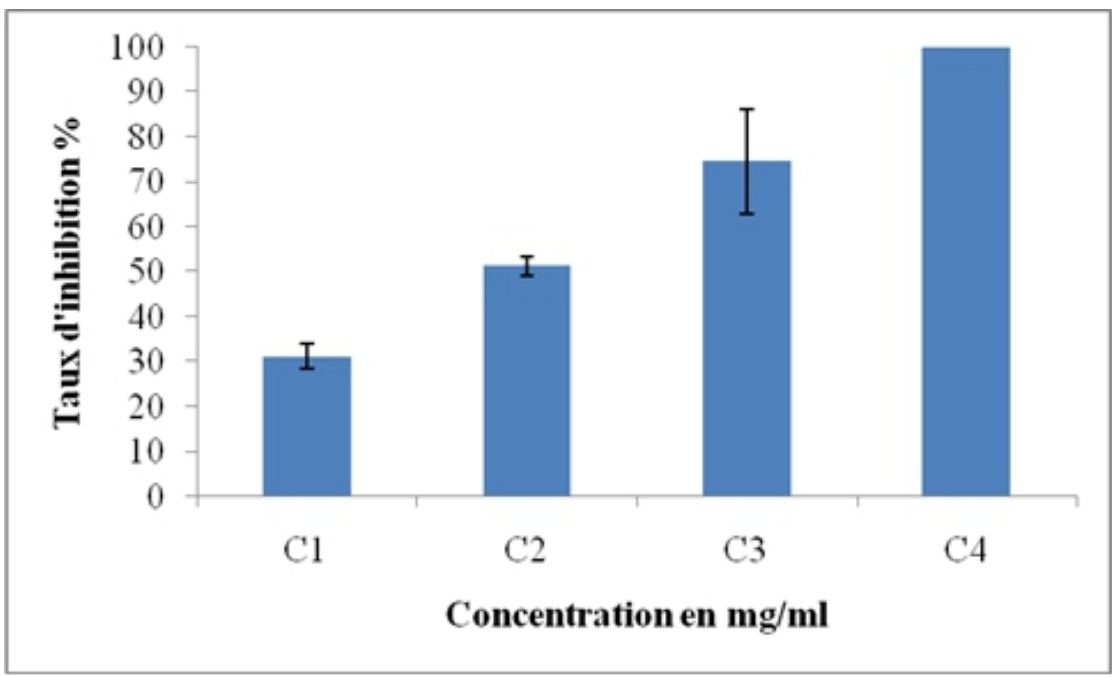

Figure 7 : Taux d'inhibition de la croissance mycélienne de Phytophthora sp. en fonction de la concentration d' $\mathrm{EE}_{13}$

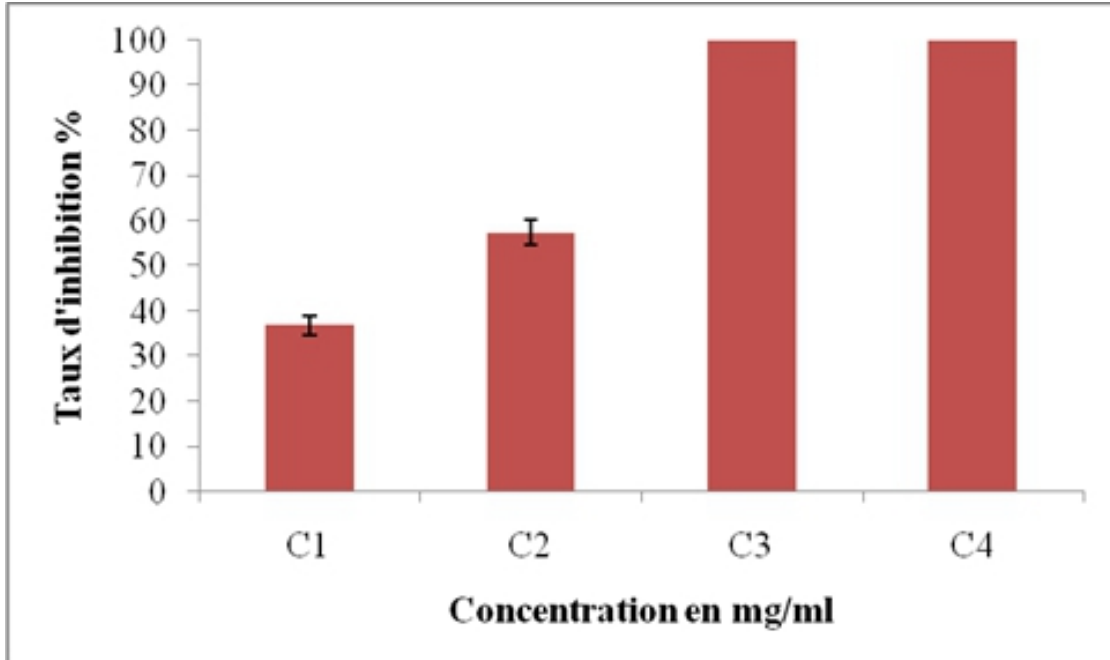

Figure 8: Taux d'inhibition de la croissance mycélienne de Fusarium sp. en fonction de la concentration d'EE 13

\section{Concentration minimales inhibitrice (CMI) et fongicide (CMF)}

$\mathrm{La}$ recherche des concentrations minimales inhibitrice (CMI) et fongicide (CMF) des extraits aqueux et éthanolique a donné les résultats consignés dans le Tableau VI. Fusarium sp et Phytophthora sp. ont eu des sensibilités différentes vis-à-vis des extraits aqueux et éthanolique d'Alchornea cordifolia. L'EE a fourni les meilleurs résultats de CMI avec 25 $\mathrm{mg} / \mathrm{ml}$ pour Fusarium $s p$. et $50 \mathrm{mg} / \mathrm{ml}$ pour Phytophthora $s p$. contre respectivement $100 \mathrm{mg} / \mathrm{ml}$ et $200 \mathrm{mg} / \mathrm{ml}$ pour l'ETA. 
Tableau VI : Concentrations minimales inhibitrice (CMI) et fongicide (CMF) des extraits aqueux et éthanolique d'Alchornea cordifolia sur Fusarium sp. et Phytophthora sp.

\begin{tabular}{|c|c|c|c|}
\hline Phytopathogène & Extrait végétal & CMI (mg/ml) & CMF (mg/ml) \\
\hline \multirow{2}{*}{ Fusarium sp. } & Extrait aqueux & 100 & 200 \\
\cline { 2 - 4 } & Extrait éthanolique & 25 & 200 \\
\hline \multirow{2}{*}{ Phytophthora sp. } & Extrait aqueux & 200 & 200 \\
\cline { 2 - 4 } & Extrait éthanolique & 50 & 200 \\
\hline
\end{tabular}

\section{Comparaison des activités antifongiques des extraits EE13 et ETA13 par le rapport d'efficacité}

Le rapport des $\mathrm{CI}_{50}$ donne $\mathrm{CI}_{50} \mathrm{ETA}_{13} / \mathrm{CI}_{50} \mathrm{EE}_{13}=26 / 10=2,6$ pour

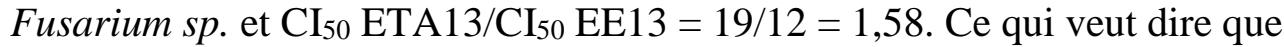
l'extrait $\mathrm{EE}_{13}$ est 2,6 fois plus actif sur Fusarium sp. que l'extrait $\mathrm{ETA}_{13}$ et 1,58 fois plus actif sur Phytophthora sp. que l'extrait ETA I13. $_{1}$.

\section{Discussion}

L'étude de l'influence des différents extraits (total aqueux et éthanolique 70\%) sur la croissance des mycéliums de Phytophthora sp. et Fusarium sp. a montré des propriétés antifongiques d'Alchornea cordifolia. Une réduction de la croissance mycélienne a été constatée parallèlement à l'augmentation de la concentration de l'extrait. Cet effet a été plus important avec l'extrait éthanolique ( $\left.\mathrm{EE}_{13}\right)$, pour lequel une inhibition de la croissance du mycélium de $50 \%$ a été obtenue à la dose de $10 \mathrm{mg} / \mathrm{ml}$ pour Fusarium $\mathrm{sp}$. et $12 \mathrm{mg} / \mathrm{ml}$ pour Phytophthora $\mathrm{sp}$. contre respectivement $26 \mathrm{mg} / \mathrm{ml}$ et 19 $\mathrm{mg} / \mathrm{ml}$ avec l'extrait aqueux (ETA13). Ces deux souches testées ont eu des sensibilités différentes vis à vis des extraits aqueux et éthanoliques. Selon le rapport des $\mathrm{CI}_{50}$ des extraits aqueux sur les extraits éthanoliques, les seconds (EE $\left.E_{13}\right)$ sont 2,6 fois plus actif sur Fusarium sp. et 1,58 fois plus actif sur Phytophthora $s p$. que les premiers (ETA 13 ). Cela s'expliquerait par le fait que les principes actifs contenus dans l'extrait éthanolique ont un plus grand potentiel antifongique. Nous en déduisons que l'éthanol permet d'extraire mieux que l'eau, les métabolites secondaires d'Alchornea cordifolia auxquels les phytopathogènes étudiés sont sensibles. Ces métabolites secondaires solubles dans l'éthanol pourraient être des terpènes, des alcaloïdes, des flavonoïdes, des iridoïdes ou des huiles végétales (Bagré et al., 2011, Goueh et al., 2015). Les analyses phytochimiques réalisées au cours de cette étude ont montré que l'extrait $\mathrm{EE}_{13}$ renferme des polyphénols, des alcaloïdes, des flavonoïdes, des polyterpènes et stérols, des tanins galliques et des catéchines mais ne contient pas de saponines ni de quinones. Quant à l'extrait ETA13, en plus de saponines présentes, celui-ci contient plus de tanins que l'extrait $\mathrm{EE}_{13}$. La différence de sensibilité des champignons vis à vis des extraits aqueux et éthanoliques pourrait se justifier par le fait que ces extraits sont composés de métabolites secondaire différents. Plusieurs travaux soutiennent que la 
majorité des métabolites secondaires des plantes sont à la base des activités physiologiques contre les microorganismes pathogènes (Adeleye et al, 2008; Mambe et al, 2016; Orsot et al., 2016; Owhe-Ureghe et al, 2016).

Le pouvoir fongicide des feuilles d'Alchornea cordifolia a été le même $(\mathrm{CMF}=200 \mathrm{mg} / \mathrm{mL}$ ) pour Fusarium sp. et pour Phytophthora sp. avec l'extrait aqueux. La concentration minimales fongicide (CMF) a été également été la même (200 mg/mL) pour Fusarium sp. et Phytophthora sp. avec l'extrait éthanolique. Ce dernier ayant la plus faible $\mathrm{CI}_{50}$ est le plus actif sur l'inhibition de la croissance Fusarium sp. que celle de Phytophthora sp. Bien que possédant de meilleurs capacités antifongiques sur Fusarium sp. par rapport aux six autres extraits, $E_{13}$ semble relativement inefficace à les tuer.

Une comparaison de l'activité de notre extrait $\mathrm{EE}_{13}$ à celle d'un antifongique de référence (Mancozèbe : $\mathrm{CI}_{50}=0,55 \mathrm{mg} / \mathrm{ml}$ ) révèle une activité antifongique très faible de $\mathrm{EE}_{13}$. En effet, ce fongicide de synthèse à large spectre a été utilisé contre Fusarium sp. À faible dose, il a exercé un pouvoir inhibiteur sur Fusarium sp. L'inhibition a été totale à partir de $3.33 \mathrm{mg} / \mathrm{ml}$ avec une $\mathrm{CI}_{90}=1,6 \mathrm{mg} / \mathrm{ml}$.

Selon la classification de Kra (2016) les CMF de nos différents extraits sont tous supérieurs à $50 \mathrm{mg} / \mathrm{ml}$, leur activité est donc très faible. Cette activité pourrait être améliorée en purifiant nos extraits par fractionnement. Par un essai bio-guidé de fractionnement liquide-liquide Manga et collaborateurs (2004) ont montré que la fraction hexanique a eu une activité nettement supérieure aux fractions aqueuses, acétate d'éthyle et hydro-insoluble lors d'une évaluation in-vivo de l'activité des feuilles d'Alchornea cordifolia.

La comparaison de nos résultats avec ceux des travaux antérieurs réalisés sur les même souches fongique par Doga (2017) montre que les extraits aqueux d'Alchornea cordifolia ont une activité antifongique plus faible que l'extrait brut aqueux de Crotalaria retusa L. qui a donné une CMF de $6.25 \mathrm{mg} / \mathrm{ml}$ pour Phytophthora sp et une CMF de $12,50 \mathrm{mg} / \mathrm{ml}$ pour Fusarium sp. Nos extraits ETA13 et $\mathrm{EE}_{13}$ sont plutôt fongistatiques que fongicides.

\section{Conclusion}

L'enquête effectuée dans le District de Yamoussoukro a permis de recenser douze plantes médicinales de la famille des Euphorbiaceae utilisées par les Baoulé. Une étude d'activité antifongique a été systématiquement effectuée avec 13 extraits aqueux d'organes issus de ces plantes à la concentration de $25 \mathrm{mg} / \mathrm{ml}$. Les trois meilleurs extraits aqueux ont été retenus pour l'évaluation de leur activité dose dépendante. Alchornea cordifolia a été retenu pour ses effets inhibiteurs aussi bien sur Phytophthora sp. que sur Fusarium sp. Le meilleur solvant a été l'éthanol qui permet une meilleure extraction des métabolites secondaires, de cette plante, actifs sur les 
phytopathogènes testés. Le pouvoir inhibiteur de l'extrait éthanolique a été plus important sur la croissance mycélienne de Fusarium sp. que Phytophthora $s p$. Ce travail préliminaire sera poursuivi par des études de fractionnement des extraits éthanoliques d'Alchornea cordifolia suivi de tests in vivo qui permettront de déterminer les concentrations efficaces et suffisantes contre Fusarium sp. et Phytophthora sp.

\section{Remerciements}

Nos remerciements vont à l'endroit des responsables des Laboratoires du Département ARA (Agriculture et Ressource Animal) de l'Institut National Polytechnique Félix Houphouët-Boigny de Yamoussoukro qui ont bien voulu mettre à notre disposition les équipements nécessaires pour la réalisation de ce travail. Ce sont en particulier les responsables et le personnel du Laboratoire de Phytopathologie et de Biologie Végétale et ceux du Laboratoire de Biotechnologie Végétale et Microbiologie Environnementale.

\section{References:}

1. Ackah J. A., 2004. Spectre anti infectieux de MISCA-F 3 sur la croissance in vitro de $C$ albicans, $T$. mentagrophytes, $T$. rubrum, $C$. neoformans, A. fumigatus et A. flavus. Mémoire de D.E.A de Biotechnologie option pharmacologie. UFR Biosciences, Université de Cocody-Abidjan, Côte d'Ivoire, 34 p.

2. Adeleye A., Ezekiel O., Smith S., Odusola O., Sobande J., 2008. Antibacterial activity of extracts of Alchornea cordifolia (Schum and Thonn) Mull.Arg., Boerhavia diffusa (L) and Bridellia micrantha (Hoscht) Baill. used in traditional medicine in Nigeria on Helicobacter pylori and four diarrhoeagenic bacterial pathogen. African Journal of Biotechnology 7(20) : 3761-3764.

3. Bagré I., Bahi C., Ouattara K., Zirihi G.N., Djaman A.J., Coulibaly A., N'Guessan J.D, 2011. Étude botanique et exploration de l'activité antifongique de Morinda morindoides (Baker) Milne-Redh. sur la croissance in vitro de Cryptococcus neoformans. Phytothérapie, 9 : $136-141$

4. De Jaeger C., Voronska E., Fraoucene N., Cherin P., 2012. Exposition chronique aux pesticides, Rôle de notre alimentation. Revue "Médecine et Longévité". Institut de médecine et physiologie de la longévité - IDJ - PARIS,Version du 22 Janvier 2018,www.institutdejager.com

5. Doga D., Zirihi G.N. et Zézé A., 2017. Propriétés antifongiques des légumineuses médicinales de Côte d'Ivoire:cas de Crotalaria retusa L. (Fabaceae) sur la croissance in vitro de Phytophthora sp. et Fusarium 
solani, deux champignons phytopathogènes. European Scientific Journal, 13(3): 371-384.

6. Dohou N., Yamni K., Badoc A. et Douira A., 2004. Activités antifongiques d'extraits de Thymelaea lythroides sur trois champignons pathogènes du riz. Bulletin de la Société de Pharmacie de Bordeaux, $143: 31-38$.

7. Flood J., 2006. The threat from global spread of cocoa pests and diseases : hypothetical scenario or clear and present danger. In: proceedings of the 15th International Cocoa Research Conference, 910 October 2006. San Jose (Costa Rica), 857-872.

8. Gnahoué G., Béné K., Coulibaly K., 2015. Etude botanique, screening phytochimique et activité anticandidosique in vitro de pycnanthus angolensis (welw.) warb. (myristicaceae). European Scientific Journal 11 (36): 1857-7881.

9. Hibar K., Medja D., Mahjoub M.E., 2007. Effect of some chemical and biological fungicides on mycelial growth and disease severity of Fusarium oxysporum $\mathrm{f}$ sp racidislycopersici. Tropicultura, 25(3): 1461452.

10. Jazet D.P.M., Tatsadjieu N.L., Tchinda S.E., Kuate J., Amvam Z.P.H. and Menut C., 2008. Antiradial potential and antifungal activities of essential oils of the leaves of Citrus latifolia against Phaeoramularia angolensis. African Journal of Biotechnology 7(22) : 4045-4050.

11. Kouadia A.M.J., 2010. Inventaire de la flore fongique des fruits frais en post récolte et test d'efficacité de quelques produits fongicides. Mémoire de fin de cycle, Diplôme Ingénieur Agronome, 63p

12. Kra A.K.M., 2016. Recherche bio guidée de composés antifongiques à partir des plantes médicinales de Côte d'Ivoire. Thèse de Doctorat de l'UFR Biosciences, Université Félix Houphouët Boigny, Abidjan, Côte d'Ivoire, $242 \mathrm{p}$.

13. Kra K.D., Diallo H.A. et Kouadio J.Y., 2009. Activités de Chromolaena odorata (L) King and Robins sur deux isolats de Fusarium oxysporum (E.F.Sm.) responsables du jaunissement mortel des feuilles de bananiers. Journal of Applied Biosciences, 24 : 1488 1496.

14. Leroux P. et Credet A., 1978. Document sur l'étude de l'activité des fongicides. INRA Versaille, France, $12 \mathrm{p}$.

15. Mambé F.T., Voukeng I.K., Beng V.P. et Kuete V., 2016. Antibacterial activities of methanol extracts from Alchornea cordifolia and four other Cameroonian plants against MDR phenotypes. Journal of Taib University, Medical Sciences, 11(2): 121-127.

16. Manga H.M., Brkic D., Marie D.E., Quetin-Leclercq J., 2004. In vivo anti-inflammatory activity of Alchornea cordifolia (Schumach. 
\&Thonn.) Mull. Arg.(Euphorbiaceae) Journal of Ethnopharmacology 92: 209-14

17. Mangambu M., Mushagalusa K., Kadima N., 2014. Contribution à l'étude phytochimique de quelques plantes médicinales antidiabétique de la ville Bukavu et ses environs (Sud-Kivu, R.D.Congo).Journal of Applied Biosciences, 75 : 6211-6220.

18. Orsot B.A.M.B. , Soro S., Konkon N.G., Kone D., Zirihi G.N., 2016. Étude ethnobotanique et évaluation in vitro de l'activité antifongique des extraits de l'écorce de Zanthoxylum gilletii sur deux souches phytopathogenes de Sclerotium rolfsii, Journal of Applied Biosciences 98: 9309-9322.

19. Owhe-Ureghe U.B., Akpo C.O., 2016. In Vitro Antibacterial Activity of Alchornea cordifolia and Costus afer Against Some Bacterial Isolates. UK Journal of Pharmaceutical and Biosciences 4(3) : 51-55.

20. Pinon A., 2000. Résidus dans les ananas en provenance d'Afrique de

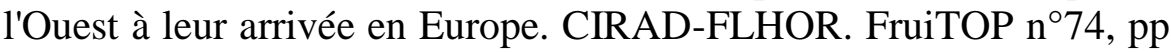
15-17.

21. Saraka A.I., Camara D., Bene K., Zirihi G.N., 2018. Enquête ethnobotanique sur les Euphorbiaceae médicinales utilisées chez les Baoulé du District de Yamoussoukro. Journal of Applied Biosciences, $126: 12734-12748$.

22. Soro D., Koné M.W., Koné D. et K. Kamanzi, 2012. Evaluation de l'activite antifongique par bioautographie de quelques plantes medicinales de côte d'ivoire contre deux formes speciales de Fusarium oxysporum. Agronomie Africaine 24(1) : 19 - 28.

23. Soro S., Abo K., Koné D., Coffi K., Kouadio J.Y. et Aké S., 2011. Comparaison de l'efficacité antifongique de l'huile essentielle d'Ocimum gratissimum $L$. et du fongicide de synthèse mancozebe contre le mycopathogène tellurique, Fusarium oxysporum f. sp en culture de tomate (Lycopersicum esculentum Mill.): Agronomie Africaine 23(1): 43 - 52.

24. Soro S., Djakalia O., Guédé Z., Coffi K., N'guessan K., Daouda K., Kouadio Y. et Aké S., 2010. Effet inhibiteur in vitro et in vivo de l'extrait de poudre et de l'huile essentielle de Xylopia aethiopica (Dunal) A. Rich. sur Fusarium oxysporium f sp. radicis-lycopersici (forl), champignon parasite des cultures de tomate. European Journal of Scientific Research, 2(39), 279-288.

25. Zirihi G.N. et Kra A.K.M., 2003. Evaluation de l'activité antifongique de Microglossa pyrifolia (Lam) O. Ktze (Asteraceae) "PYMI" sur la croissance in vitro de candida albicans. Revue médicale et Pharmacopée Africaine, 17: 11-18. 